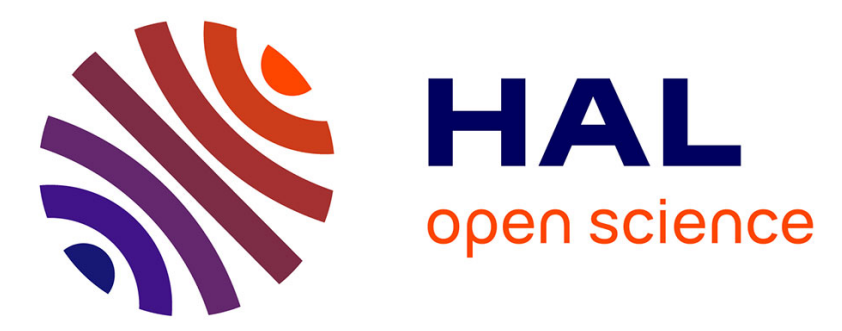

\title{
Formation of Substitutional-Interstitial Clusters in Dilute Alloys: Development of Previous Models
}

\author{
A. Biscarini, R. Campanella, B. Coluzzi, F. Mazzolai
}

\section{To cite this version:}

A. Biscarini, R. Campanella, B. Coluzzi, F. Mazzolai. Formation of Substitutional-Interstitial Clusters in Dilute Alloys: Development of Previous Models. Journal de Physique IV Proceedings, 1996, 06 (C8), pp.C8-51-C8-54. 10.1051/jp4:1996809 . jpa-00254526

\section{HAL Id: jpa-00254526 https://hal.science/jpa-00254526}

Submitted on 1 Jan 1996

HAL is a multi-disciplinary open access archive for the deposit and dissemination of scientific research documents, whether they are published or not. The documents may come from teaching and research institutions in France or abroad, or from public or private research centers.
L'archive ouverte pluridisciplinaire HAL, est destinée au dépôt et à la diffusion de documents scientifiques de niveau recherche, publiés ou non, émanant des établissements d'enseignement et de recherche français ou étrangers, des laboratoires publics ou privés. 


\title{
Formation of Substitutional-Interstitial Clusters in Dilute Alloys: Development of Previous Models
}

\author{
A. Biscarini, R. Campanella, B. Coluzzi and F.M. Mazzolai \\ Department of Physics, University of Perugia, Via A. Pascoli 5, 06100 Perugia, Italy
}

\begin{abstract}
The anelastic behaviour of crystalline solids containing light interstitials and trapping substitutional impurities has been theoretically investigated. New formulas have been derived which allow the calculation of the concentration of substitutional-interstitial (SI) clusters as a function of the overall contents of substitutional and interstitial impurities. The formation of higher order complexes such as $\mathrm{SI}_{\mathbf{n}}$ is also considered. The effect of shortrange repulsive interactions among the interstitials is taken into account in the framework of the hard-sphere model.
\end{abstract}

\section{INTRODUCTION}

It is well known that the presence of a small amount of substitutional alloying elements considerably affects the anelastic behaviour of crystalline solids containing light interstitials [1]. Approximate expressions for the equilibrium concentration of the interstitial impurities over the available interstitial sites have been derived by Hohler and Kronmüller [2] and by Zapp and Birnbaum [3]. A more general treatment has been given by Cannelli et al. [4] through the application of the grand canonical distribution, in the assumption of a noninteracting gas of interstitials. The aim of the present work has been to develop a new approach in the formalism of the canonical ensemble that might take into account short-range repuslive interactions among the interstitials.

\section{STATISTICAL MODEL}

Le us consider a crystal containing $\mathrm{N}_{S}$ substitutionals (S) and $\mathrm{N}_{\mathrm{I}}$ interstitials (I). We are primarily interested in the localization of I among the available interstitial sites under the following assumptions:

1) the I occupy only one kind of sites (octahedral or tetrahedral). For the case of $\mathrm{H}$ interstitials, tetrahedral sites are supposed to be occupied in bcc lattices and octahedral sites in fcc lattices;

2) an $S$ atom only interacts with the I (bound I) occupying one of the $z$ interstitial sites nearest neighbour to the $\mathrm{S}$ (bound sites);

3) all the substitutionals are immobile and isolated in the host metal matrix in such a way not to share bound sites, whose number $N_{B}$ is, thus, given by $N_{B}=z N_{S}$. This should be a good approximation for a low concentration of $S$;

4) no clustering of unbound I occurs;

5) substitutional-interstitials complexes $S I_{j}$ of energy $E_{j}$ are identified only on the basis of the number $j$ $(j=1,2, \ldots, m ; m \leq z)$ of bound $I$. The possibility that some of the higher-order complexes may not exist is also considered.

\subsection{Absence of interactions among interstitials}

At first, the assumption will be made that only interactions among I belonging to the same $S I_{j}$ complex exist. Denoting by $n_{0}$ the number of unbound $I$ and by $n_{j}(\mathrm{j}=1,2, \ldots, \mathrm{m})$ that of $S I_{j}$ complexes, each state of 
the system is specified by a set of "occupation numbers" $\left\{n_{j}\right\}=n_{0}, n_{1}, \ldots, n_{m}$. The energy of the system in a $\left\{n_{j}\right\}$ state is then given by

$$
E\left\{n_{j}\right\}=\sum_{j=0}^{m} n_{j} E_{j}=N_{I} E_{0}-\sum_{j=1}^{m} n_{j} B_{j}
$$

where $E_{0}$ is the energy of an unbound I, $E_{j}$ is the energy of a $S I_{j}$ complex, and $B_{j}=j E_{0}-E_{j}$ the binding energy of such a complex. If $B_{1}>O\left(B_{1}<0\right)$ the $\mathrm{S}$ atom is a trapping (antitrapping) impurity; $B_{j}=0$ $(\mathrm{j}=1,2, \ldots, \mathrm{m})$ defines a completely random solid solution of interstitials. The thermodynamic weight $\mathrm{W}\left\{n_{j}\right\}$ of the $\left\{n_{j}\right\}$ state is found to be given by:

$$
W\left\{n_{j}\right\}=W_{0} \frac{N_{S} !}{\left(N_{S}-\sum_{j=1}^{m} n_{j}\right) !} \prod_{j=1}^{m} \frac{1}{n_{j} !}\left(\begin{array}{c}
z \\
j
\end{array}\right)^{n_{j}} ; \quad W_{0}=\left(\begin{array}{c}
N_{0} \\
n_{0}
\end{array}\right)
$$

where $\mathrm{N}_{0}$ is the number of unbound sites. With the help of the Stirling's formula the entropy $S\left\{n_{j}\right\} \equiv k_{B} l n$ $W\left\{n_{j}\right\}$ is found to satisfy the relation

$$
\frac{S\left\{n_{j}\right\}-S\{0\}}{k_{B}} \cong \sum_{j=0}^{m}\left[n_{j} \ln \left(\begin{array}{c}
z \\
j
\end{array}\right)-n_{j} \ln n_{j}\right]-\left(N_{0}-n_{0}\right) \ln \left(N_{0}-n_{0}\right)-\left(N_{S}-\sum_{j=1}^{m} n_{j}\right) \ln \left(N_{S}-\sum_{j=1}^{m} n_{j}\right)
$$

In the canonical distribution the probability of $\left\{n_{j}\right\}$ state is given by $\operatorname{Pr}\left\{n_{j}\right\}=Z^{-1} W\left\{n_{j}\right\} \exp \left\{-\beta E\left\{n_{j}\right\}\right]$, where $Z=\Sigma_{\left\{n_{j}\right\}} W\left\{n_{j}\right\} \exp \left(-\beta E\left\{n_{j}\right\}\right)$ is the canonical partition function and $\beta=1 /\left(k_{B} T\right)$. The set of numbers $\left\{n_{j}\right\}$ which maximize this probability (most probable values) are the same as those which minimize the Helmholtz free energy $F\left\{n_{j}\right\}=E\left\{n_{j}\right\}-T S\left\{n_{j}\right\}=-k_{B} T \ln \left[W\left\{n_{j}\right\} \exp \left(-\beta E\left\{n_{j}\right\}\right)\right]$. Since the number $N_{I}$ of I is given, the following constraint is to be imposed to the variable $\left\{n_{j}\right\}$

$$
N_{I}=n_{0}+\sum_{j=1}^{m} j n_{j}
$$

The most probable values of $\left\{n_{j}\right\}$ are to be determined under condition (4) by solving the $\mathrm{m}+1$ equations $\left(\partial F / \partial n_{j}\right)+\mu\left(\partial N_{I} / \partial h_{j}\right)=0 \quad(j=0,1, \ldots, m)$, where $\mu$ is a Lagrange multiplier. With the help of the previous relations these equations can be written as:

$$
\begin{gathered}
n_{0}=\frac{N_{0}}{e^{\beta\left(E_{0}-\mu\right)}+1} \\
n_{j}=\frac{\left(\begin{array}{c}
z \\
j
\end{array}\right)\left(N_{S}-\sum_{k=1}^{m} n_{k}\right)+n_{j}}{e^{j \beta\left(\varepsilon_{j}-\mu\right)}+1} \quad j=1,2, \ldots, m
\end{gathered}
$$

Since $N_{S}-\sum_{k=1}^{m} n_{k}$ is the number of $S$ with no bound I and $\left(\begin{array}{c}z \\ j\end{array}\right)$ that of the different configurations for a $S I_{j}$ complex, the numerator in $(5 \mathrm{~b})$ represents the number of configurations which are either occupied or can be occupied by a $S I_{j}$ complex. The solution of the $\mathrm{m}+2$ equations (4), (5a) and (5b) determines the $\mathrm{m}+2$ variables $\mu$ and $\left\{n_{j}\right\}$. To solve the system numerically it is useful to rewrite eq.s $(5 \mathrm{~b})$ in the form

$$
n_{j}=N_{S}\left[\sum_{j=0}^{m}\left(\begin{array}{l}
z \\
j
\end{array}\right) e^{-\beta\left(E_{j}-j \mu\right)}\right]^{-1}\left(\begin{array}{l}
z \\
j
\end{array}\right) e^{-\beta\left(E_{j}-j \mu\right)} \quad j=1,2, \ldots, m
$$


which is also deducible by application of the gran canonical distribution to the set of $z$ interstitial sites coordinated to a S atom, assuming this set is in contact with a source of I characterized by the chemical potential $\mu$ [4]. Eq.s (6) give the $\left\{n_{j}\right\}$ when $\mu$ is used as a parameter to satisfy condition (4). The parameter $\mu$ may be eliminated from equations (5a) and (5b) to give the following "laws of mass action"

$$
\frac{n_{j}\left(M-Z N_{S}-n_{0}\right)^{j}}{\left(\begin{array}{l}
z \\
j
\end{array}\right)\left(N_{S}-\sum_{j=1}^{m} n_{j}\right) n_{0}^{j}}=e^{B_{j} / k_{B} T} \quad j=1, \ldots, m
$$

which describe the formation (dissolution) of the complex $S I_{j}$ from (in) $\mathrm{j}$ unbound $\mathrm{I}$. For $\mathrm{m}=1$, that is, for the only formation of complexes of order one $(S I)$, the atomic concentration $c_{S I} \quad\left(c_{S I}=n_{1} / N_{M e} ; N_{M e}=\right.$ number of metal atoms) of SI pairs may be deduced from (7) as a function of the binding energy $B_{l}$ and the overall concentration of $\mathrm{I}\left(c_{I}=N_{I} / N_{M e}\right)$ and $\mathrm{S}\left(c_{S}=N_{S} / N_{m e}\right)$ according to the relation

$$
c_{S I}=\frac{(1-\lambda) c_{1}+(1-z \lambda) c_{S}+\lambda \gamma-\sqrt{\left[(1-\lambda) c_{1}+(1-z \lambda) c_{S}+\lambda \gamma\right]^{2}-4(1-\lambda) c_{1} c_{S}}}{2(1-\lambda)}
$$

where $\lambda=\exp \left(-B / k_{B} T\right) / z$, and $\gamma$ is the ratio of the overall number of interstitial site to the number of metal atoms $\left(N_{M e}\right)$. In figure 1 and 2 the ratio $c_{S I} / c_{S}$ is plotted for different values of the binding energy $B_{I}$ as a function of the normalized interstitial concentration $c_{T} / c_{S}$ and the inverse of the temperature $T^{-1}$, respectively. From equation (7) for $\mathrm{m}=\mathrm{j}=1$ the approximated formulas for $c_{S I}$ given by Hohler and Kronmüller [2] and Zapp and Birnbaum [3] are easily deduced.

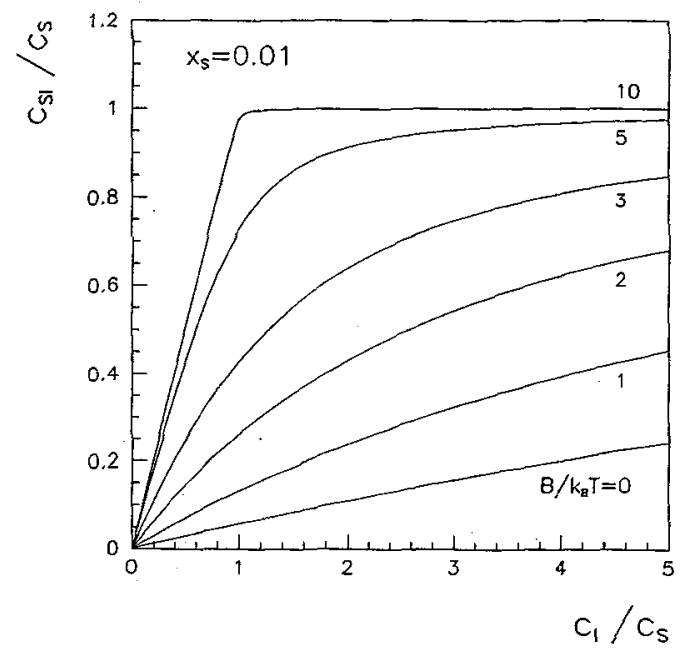

Figure 1: Dependence of the concentration of the SI complexes on the concentration of the $I$ impurities for different values of $B / k_{B} T$ ( $B=$ binding energy)

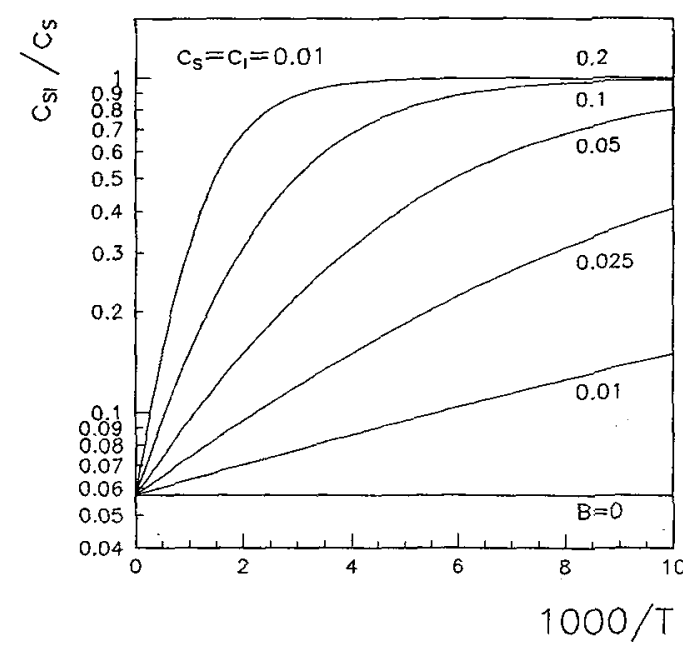

Figure 2: Dependence of the concentration of the SI complexes on $T$ for different values of the binding energy $B$ in $\mathrm{eV}$

\subsection{Short-range repulsive interaction among interstitials}

Short-range repulsive interactions among unbound interstitials will be taken into account within the framework of the hard core model. Each unbound I is assumed to prevent occupancy by onther I of a certain number of its neighbouring unbound sites (blocked sites). For $\mathrm{H}$ interstitials a blocking distance of about $0.21 \mathrm{~nm}$ from an occupied site is to be taken [5]. Let $G(j)$ be the number of sites blocked when $j$ unbound sites are occupied. The thermodynamic weight is again expressed by (2) with $W_{0}$ given by 


$$
W_{0}=\left[N_{0}\right]\left[N_{0}-1-G(1)\right] . .\left[N_{0}-\left(n_{0}-1\right)-G\left(n_{0}-1\right)\right] / n_{0} !
$$

The contribution of unbound I to the entropy of the system is propotional to [6]

$$
\frac{\ln W_{0}}{N_{0}}=-x_{0} \ln x_{0}+x_{0}+\int_{0}^{x_{0}} \ln [1-\xi-g(\xi)] d \xi
$$

where $x_{0}=n_{0} / N_{0}, \xi=j / N_{0}, g=G / N_{0}$. From the equalities

$$
\frac{d}{d n_{0}}\left(\ln W_{0}\right)=\frac{d}{d x_{0}}\left(\frac{\ln W_{0}}{N_{0}}\right)=-\ln \frac{x_{0}}{1-x_{0}-g\left(x_{0}\right)}=-\ln \frac{n_{0}}{N_{0}-n_{0}-G\left(n_{0}\right)}
$$

the effect of blocking among unbound I reduces to replace eq. (5a) with

$$
n_{0}=\frac{N_{0}-G\left(n_{0}\right)}{e^{\beta\left(E_{0}-\mu\right)}+1}
$$

that is to replace the number $N_{0}$ of unbound sites with that $N_{0}-G\left(n_{0}\right)$ of unblocked and unbound sites. Approximate formulas for $G$ have been derived by Boureau for tetrahedral site occupancy in bcc lattices by assuming blocking of sites up to the third nearest neighbours to an interstitial [7]. When blocking of bound sites by bound atoms is also taken into account, the binomial coefficient $\left(\begin{array}{c}Z \\ j\end{array}\right)$ is to be substituted with $[z][z-1-G(1)] \ldots[z-(j-1)-G(j-1)] / j !$ in the equations (5b). The blocking effects of unbound sites by bound and viceversa, which are negligible in diluite alloys have been evaluated for the case of concentrated alloys in previous papers $[8,9]$.

\section{ANELASTIC RELAXATION DUE TO SI COMPLEXES}

The previous calculations can be used for a qualitative interpretation of relaxations due to stress-induced intersite transitions of $\mathrm{H}$ impurities in metal systems containing a low concentration of S impurities [10]. Different effects can be distinguished according to the nature (bound, unbound) of the sites involved in the jump: intersite transitions of unbound interstitials (Snoek effect), reorientations of $S I_{j}$ complexes, formation/dissolution of a complex of order $\mathrm{j}$ from/into a complex of order $\mathrm{j}-1: S i_{j}=S I_{j-1}+I \quad(\mathrm{j}=1,2 \ldots, \mathrm{m})$. When the concentration of impurities become appreciable, long range S-I and I-I interactions and lattice dilation cannot be neglected. These effects have been qualitatively accounted for by the addition of a I atoms concentration dependent term $f\left(N_{j}\right)$ to the site energies $E_{j}[11]$ or by spreading the energy levels $E_{j}$ in a finite-width Gaussian or Lorentzian-distributed bands of energies [4]. A quantitative estimate of the damping spectrum requires the knowledge of the numerical values of principal componentes of the elastic dipole tensor related to the different defect species as well as the relaxation parameters (frequency factor and activation energy) of each elementary jump process [1].

\section{References}

[1] Nowick A.S., Berry B.S., Anelastic Relaxation in Cristalline Solids (Pergamon Press, New York, 1972)

[2] Hohler B., Kronmüller H., Z. Phys. Chem. N. F. 114, (1979) S.93-108

[3] Zapp P.E., Birnbaum H. K., Acta Met. 28, (1979) 1275

[4] Cannelli G., Cantelli R., Cordero F., Phys. Rev. B 32 (1985) 3573

[5] Switendick A., Z. Phys. Chem N. F. 117, (1979) 89

[6] Thorn R. J., Winslow G. H., J. Chem. Phys. 44 (1965) 2632

[7] Boureau C., J. Phys. Chem. Solids 42 (1981) 743

[8] Biscarini A., Coluzzi B., Mazzolai F.M., Phys. Rev. B 49 (1993) 969

[9] Biscarini A., Coluzzi B., Mazzolai F.M., submitted to Phys. Rev. $B$.

[10] Coluzzi B., Biscarini A., Campanella R., F.M.Mazzolai, to be published

[11] Brouwer R.C., Griessen R., Phys. Rev. B 40 (1989) 3546 